\title{
Integración de recursos audiovisuales y multimedia como objetos de aprendizaje en escuelas públicas de Costa Rica con acceso a computadoras XO
}

Integration of audiovisual and multimedia resources as learning objects in Costa Rican public schools with acces to XO computers

\author{
Volumen 17, Número 1 \\ Enero-Abril \\ pp. 1-29
}

Este número se publicó el $1^{\circ}$ de enero de 2017

DOI: http://dx.doi.org/10.15517/aie.v17i1.27454

Aarón Mena Araya

Revista indizada en REDALYC, $\underline{\text { SCIELO }}$

Revista distribuida en las bases de datos:

LATINDEX, DOAJ, REDIB, IRESIE, CLASE, DIALNET, SHERPA/ROMEO, QUALIS-CAPES, MIAR

Revista registrada en los directorios:

ULRICH'S, REDIE, RINACE, OEI, MAESTROTECA, PREAL, CLACSO 


\title{
Integración de recursos audiovisuales y multimedia como objetos de aprendizaje en escuelas públicas de Costa Rica con acceso a computadoras XO
}

Integration of audiovisual and multimedia resources as learning objects in Costa Rican public schools with acces to XO computers

\begin{abstract}
Aáron Mena Araya ${ }^{1}$
Resumen: El Ministerio de Educación Pública de Costa Rica y la Fundación Quirós Tanzi implementan desde el 2012 el Proyecto Conectándonos, a través del cual distribuyen computadoras XO a escuelas primarias en un modelo uno a uno. La información disponible sobre la forma en que las personas docentes integran los recursos audiovisuales y multimedia a las actividades de aprendizaje mediadas con las computadoras XO es limitada. Con el objetivo de conocer si las computadoras XO han facilitado la utilización de objetos de aprendizaje, y de obtener insumos para el diseño de recursos audiovisuales y multimedia que puedan ser aprovechados en las escuelas del Proyecto, se aplicó una encuesta a 186 personas vinculadas al Proyecto Conectándonos. Fue posible determinar que 82.7 \% de las personas docentes emplean las computadoras XO en clase al menos una vez a la semana y que las asignaturas en las que menos se usan son inglés y matemática. Asimismo, se determinó que el profesorado utiliza regularmente recursos audiovisuales y multimedia, pero en la mayoría de los casos estos no son aprovechados como objetos de aprendizaje. Esto significa que los recursos no son manipulados, modificados ni creados por el cuerpo estudiantil. Este documento presenta los resultados de la encuesta, elabora sobre la construcción conceptual de objeto de aprendizaje y sugiere el proceso de investigación, diseño y producción de dos paquetes de recursos audiovisuales y multimedia, para ser empleados como objetos de aprendizaje en las asignaturas de matemática e inglés, aprovechando las posibilidades del modelo uno a uno de las escuelas del Proyecto Conectándonos.
\end{abstract}

Palabras clave: objeto de aprendizaje, computadora, audiovisual, multimedia

Abstract: The Ministry of Public Education of Costa Rica and Quiros Tanzi Foundation have implemented since 2012 the Conectandonos Project, through which XO computers are distributed to primary schools in a one to one computing education model. Available information on how teachers incorporate audiovisual and multimedia resources to learning activities mediated by XO computers is scarce. With the aim of finding out if XO computers have boosted the use of learning objects and collecting information for the designing of audiovisual and multimedia resources, a survey was applied to 186 teachers working with Conectandonos Project. It was determined that $82.7 \%$ of the teachers use XO computers in their classes at least once a week, and that English and Mathematics are the subjects in which computers are used the least. It was also found that even though teachers regularly use audiovisual and multimedia resources, in most cases these are not utilized as learning objects. This means resources are rarely manipulated, modified or created by the students. This document elaborates on the construction of the concept of learning object, and describes the research, design and production processes involved in the creation of two audiovisual and multimedia resources bundles to be used as learning objects in English and Mathematics classes, taking advantage of the possibilities of Conectandonos Project one-to-one computing education model.

Keywords: learning object, computer, audiovisual, multimedia

\footnotetext{
${ }^{1}$ Máster en Ciencias de la Cultura, Universidad de Ibaraki, Japón. Profesor Asociado de la Universidad de Costa Rica. . Investigador en el Centro de Investigación en Comunicación de la Universidad de Costa Rica.
}

Dirección electrónica: AARON.MENAARAYA@ucr.ac.cr

Artículo recibido: 4 de marzo, 2016

Enviado a corrección: 19 de octubre, 2016

Aprobado: 05 de diciembre, 2016 


\section{Introducción}

Las computadoras XO fueron diseñadas por la Fundación One Laptop Per Child (OLPC), como un dispositivo digital de bajo costo que pudiera ser integrado en los procesos de educación primaria de los países en vías de desarrollo. Las computadoras XO pueden ser conectadas a internet vía wifi (sistema para el envío inalámbrico de datos dentro de una red de computadoras), y con otras computadoras a través de una red mesh (red local en la que los dispositivos se encuentran conectados directamente entre sí), para compartir archivos y realizar actividades de aprendizaje de manera colaborativa. Adicionalmente, utilizan sistemas operativos y aplicaciones de software libre, lo cual reduce su costo de compra y operación.

La distribución de las computadoras XO en el mundo inició en el 2007, superando los 2,5 millones de unidades para el 2014. En Latinoamérica, nueve países (México, Guatemala, Nicaragua, Costa Rica, Colombia, Perú, Paraguay, Uruguay y Argentina) han implementado programas para la distribución y aprovechamiento de computadoras $\mathrm{XO}$, entre los que sobresale el Plan Ceibal. Este programa fue desarrollado por el gobierno de Uruguay entre el 2007 y el 2009, y ostenta el logro inédito de gestionar la distribución de los dispositivos a la totalidad de las escuelas primarias, incidiendo significativamente en la disminución de las brechas de acceso al internet y a las tecnologías digitales. (Administración Nacional de Educación Pública, 2011)

En Costa Rica, la Fundación Quirós Tanzi y el Departamento de Recursos Tecnológicos en Educación del Ministerio de Educación Pública implementan desde el 2012 el Proyecto Conectándonos, a través del cual se distribuyen computadoras XO en escuelas primarias públicas, de manera que estas puedan ser incorporadas a las actividades de enseñanza y aprendizaje en las aulas, mediante un modelo uno a uno. En este modelo cada niño y niña recibe una computadora que puede usar tanto en el aula bajo la guía de la persona docente, como en el hogar, en actividades de aprendizaje individual. De este modo, mediante el Proyecto Conectándonos se crean condiciones inéditas en las escuelas públicas del país, para la creación de ambientes de aprendizaje mediados con tecnologías digitales.

El Proyecto inició en el 2012 con 15 escuelas públicas, vinculando a 1523 estudiantes y 120 docentes. Para el 2015, la cobertura del Proyecto se extendió a 64 escuelas, 4640 estudiantes y 510 docentes. Es importante mencionar que en el 2016 Costa Rica tuvo una población estimada de 4890372 personas (Instituto Nacional de Estadística y Censos, 2016), distribuidas en un área de 51100 kilómetros cuadrados, y que para inicios de 2015 
contaba con 4107 escuelas primarias (3780 públicas y 327 privadas) en las que estudiaban 443683 niños y niñas. (Ministerio de Educación Pública, 2016)

En este contexto, se reconoció la necesidad de realizar un acercamiento a la manera en que las personas docentes de las escuelas del Proyecto Conectándonos aprovechan las computadoras XO para integrar los recursos audiovisuales y multimedia al trabajo en el aula. Dicho acercamiento se llevó a cabo mediante los proyectos de investigación "Posibilidades para la producción de recursos audiovisuales educativos para ser utilizados en las Computadoras XO en las escuelas públicas del país" y "Producción de recursos audiovisuales para favorecer el desarrollo de las competencias ATC21S en las escuelas con acceso a computadoras XO u otras tecnologías que permitan la utilización de audiovisuales en el aula", inscritos en el Centro de Investigación en Comunicación (CICOM) de la Universidad de Costa Rica.

Este documento propone una definición operativa de objeto de aprendizaje, presenta los resultados de una encuesta aplicada a las personas docentes del Proyecto Conectándonos, y describe las actividades de investigación y acción social realizadas para atender las necesidades identificadas.

\section{Objetos y objetivos de aprendizaje}

EI Proyecto Conectándonos presenta "una propuesta pedagógica fundamentada en la visión filosófica constructivista" (Ministerio de Educación Pública de Costa Rica y Fundación Quirós Tanzi, 2014, p. 4), en la cual se articula la visión de Seymour Papert.

Papert propuso el construccionismo como un abordaje teórico a la utilización de las tecnologías digitales en la educación. En este abordaje, se le otorga a cada estudiante un rol activo, en el que puede diseñar sus propios proyectos y construir su propio aprendizaje. (Badilla y Chacón, 2004, p.2-4)

Papert hace énfasis en la necesidad de crear "objetos para pensar", que puedan ser aprovechados en ambientes de aprendizaje mediado por computadora. Plantea que estos objetos pueden ser utilizados para pensar sobre otras cosas mediante una construcción propia de los mismos, y que las personas crean su propio entendimiento del mundo al crear artefactos, modificarlos y ver cómo funcionan. (Badilla y Chacón, 2004, p.6)

Con el fin de propiciar ambientes de aprendizaje construccionistas, el teórico desarrolló junto con sus colaboradores lenguajes de programación, dirigidos a facilitar el aprendizaje intuitivo de la geometría y las matemática. Estas herramientas fueron incorporadas a partir 
de los años ochenta en espacios de educación primaria de distintos países del mundo, entre ellos Costa Rica.

La propuesta pedagógica del Proyecto Conectándonos enfatiza la dimensión social del aprendizaje y el aprovechamiento de las nuevas tecnologías, lo cual se ve reflejado en dos de sus objetivos específicos (Proyecto Conectándonos, 2012, p.8):

- Potenciar en los niños el desarrollo de la fluidez tecnológica y las competencias de aprendizaje colaborativo y resolución de problemas, en ambientes de aprendizaje cambiantes.

- Desarrollar un programa de formación profesional docente, que empodere a los maestros para que sean mediadores del proceso de enseñanza-aprendizaje, acorde con la propuesta pedagógica construccionista del proyecto.

Adicionalmente, la propuesta identifica a las competencias ATC21S (Griffin, McGraw y Care, 2012), como un marco de referencia para el desarrollo transversal de conocimientos, habilidades, actitudes, éticas y valores útiles en la sociedad de la información y el conocimiento. Es importante mencionar que cuatro de las competencias ATC21S guardan relación directa con los elementos planteados en los objetivos del Proyecto Conectándonos: pensamiento crítico, resolución de problemas y toma de decisiones (correspondiente a la categoría de maneras de pensar), colaboración (correspondiente a la categoría de maneras de trabajar), alfabetización en información y en tecnologías de la información y la comunicación (correspondientes a la categoría de herramientas para trabajar).

El marco ATC21S también incluye las competencias de creatividad e innovación, aprendiendo a aprender o metacognición (maneras de pensar), comunicación (maneras de trabajar), ciudadanía local y global, vida y carrera, responsabilidad personal y social (maneras de vivir en el mundo).

Por una parte, el personal de apoyo pedagógico del Ministerio de Educación Pública y la Fundación Quirós Tanzi introducen a las personas docentes con una capacitación inicial de una semana, al final de la cual se les entregan las computadoras XO. Posteriormente, personas asesoras pedagógicas de la Fundación Quirós Tanzi realizan distintos tipos de acompañamiento a las personas docentes participantes en el Proyecto. Uno de estos seguimientos se denomina Couching, y consiste en visitas bisemanales en las cuales la persona asesora observa el trabajo de los docentes y le realiza recomendaciones de actividades de aprendizaje mediadas por la computadora XO. La regularidad de las visitas pasa a ser mensual a partir del segundo año de integración al Proyecto. (Mena, 2015, p.173) 
Por otra parte, el mismo personal de la Fundación Quirós Tanzi realiza talleres cada cuatro o seis semanas, en los que se profundizan los elementos teóricos y técnicos introducidos en la capacitación inicial. En las escuelas en las que ya se han impartido los distintos talleres y se reconoce un adecuado apropiamiento de la computadora por parte de docentes y estudiantes, se recurre a la modalidad de acompañamiento a distancia. Este acompañamiento utiliza espacios en redes sociales, correo electrónico y mensajería telefónica para compartir sugerencias de actividades de aprendizaje y recordatorios sobre las posibilidades de las distintas herramientas con las que cuenta la computadora XO. (Mena, 2015, p.174)

Es importante mencionar que las capacitaciones se enfocan en la creación de ambientes de aprendizaje constructivistas y en el apoyo a la persona docente en el planeamiento de actividades de que aprovechen las computadoras XO en la utilización de objetos de aprendizaje. Los proyectos de aprendizaje realizados en las aulas buscan incidir en el desarrollo de habilidades de indagación, investigación y razonamiento, centrados en una colaboración articulada en el uso de las tecnologías digitales (Ballestero, 2012, p.31).

Esta investigación explora el empleo de recursos audiovisuales y multimedia en el contexto del Proyecto Conectándonos, brindando especial atención a su dimensión de objeto de aprendizaje.

\subsection{Objetos de aprendizaje}

La discusión sobre lo que puede considerarse un objeto de aprendizaje se ha alimentado de distintas perspectivas, permaneciendo como un elemento central las consideraciones sobre la inclusión de objetos digitales o estrictamente vinculados a las ciencias informáticas, y de objetos tangibles o analógicos. Los objetos de aprendizaje fueron comprendidos inicialmente como elementos de instrucción mediada por computadora, que podían ser creados por las personas a cargo de los procesos de instrucción, y reutilizados en diversos contextos de aprendizaje (Dahl y Nygaard, 1966). Reconociendo un espectro más amplio, Wiley (2000) propone que los objetos de aprendizaje incluyen a cualquier recurso digital que pueda ser reutilizado en el apoyo al aprendizaje.

Por un lado, esta delimitación del objeto de aprendizaje fue expandida nuevamente en la definición propuesta por el Instituto de Ingenieros Eléctricos y Electrónicos de Nueva York, la cual los comprende como cualquier entidad, digital o no digital, que puede ser utilizada, reutilizada y referenciada durante el aprendizaje apoyado con tecnologías (IEEE, 2002, p.4). Dentro de esta definición pueden ser incluidos recursos no digitales, como materiales 
didácticos impresos en láminas transparentes y presentadas con un retroproyector. Del mismo modo, pueden ser incluidos recursos digitales de audio y video. (Ritzhaupt, 2010, p.218).

Por otro lado, el Ministerio de Educación Pública de la República de Colombia (2012) elabora sobre la estructura de los objetos de aprendizaje, y señala que en el contexto de los ambientes de aprendizaje mediados con nuevas tecnologías, estos pueden comprenderse como recursos digitales reutilizables, con un propósito educativo y constituidos por al menos tres componentes internos: contenidos, actividades de aprendizaje y elementos de contextualización.

Mientras los contenidos corresponden a representaciones de información orientadas al aprendizaje de conocimientos, habilidades o actitudes, y las actividades de aprendizaje corresponden a propuestas de trabajo elaboradas por el personal docente con el objetivo de que cada estudiante adquiera y desarrolle dichos conocimientos, habilidades y actitudes, los elementos de contextualización engloban la información que permiten la búsqueda y selección de un objeto de aprendizaje adecuado para satisfacer una necesidad educativa específica. Los elementos de contextualización pueden incluir resúmenes, objetivos de aprendizaje y preguntas generadoras.

Tomando en consideración los objetivos del Proyecto Conectándonos y las condiciones específicas de la educación primaria pública de Costa Rica, el autor de este artículo propone la siguiente definición operativa de objeto de aprendizaje: un recurso físico o digital recopilado, modificado o creado por la persona docente para ser puesto a disposición del estudiantado, quien se apropia de dicho recurso, ya sea modificándolo, ampliándolo o creando recursos nuevos a partir de él, y lo utiliza como una herramienta para el intercambio dialógico de conocimientos, habilidades y actitudes, en colaboración con sus pares y con la persona docente.

Vale mencionar que los objetos de aprendizaje también pueden comprenderse desde una relación dicotómica con los "objetos de enseñanza", los cuales corresponden a recursos físicos o digitales creados, recopilados o modificados por la persona docente para luego administrarlo de manera exclusiva, en la transmisión unidireccional de conocimientos, habilidades y actitudes. De este modo, los objetos de enseñanza engloban a todos aquellos recursos que la persona docente utiliza para organizar y presentar información, y que se encuentran insertos en procesos de enseñanza en los que las personas estudiantes cumplen un rol estrictamente pasivo. 
En los procesos de educación formal, la construcción de objetivos de aprendizaje cumple una función central en la delimitación de los objetos de estudio, y en la definición de los perfiles que se busca sean adquiridos por las personas estudiantes. La inclusión de objetos de aprendizaje a la dinámica de las aulas hace necesario el planteamiento de objetivos de aprendizaje que tomen en consideración el tipo de actividades en los que dichos objetos efectivamente pueden ser aprovechados en la construcción de ambientes de aprendizaje constructivistas, y que puedan además contribuir al desarrollo de competencias como las propuestas en el marco ATC21S.

Bloom (1956) propone una taxonomía con seis categorías para las dimensiones cognitivas que pueden ser afectadas mediante la participación en actividades de aprendizaje y el contacto con recursos didácticos: conocimiento, comprensión, aplicación, análisis, síntesis y evaluación. La descripción de estos dominios sirve de guía para comprender y delimitar la incidencia de una actividad de aprendizaje específica, y perfilar los objetivos de dicha actividad.

Tomando en consideración la necesidad de ampliar el rango de procesos cognitivos que pueden ser identificados para la definición de objetivos de aprendizaje, y las nuevas posibilidades facilitadas por la inclusión de las tecnologías informáticas a las dinámicas de enseñanza y aprendizaje, Krahwohl plantea una revisión de la taxonomía de Bloom, en la que entre otros cambios, se replantea la dimensión de síntesis, para dar forma a la dimensión de "crear" (Krathwohl, 2002, p. 214). La tabla No.1 presenta las categorías presentadas en la revisión de Krathwohl, y los procesos cognitivos involucrados con cada una de estas categorías.

Tabla No.1

Taxonomía de Bloom revisada por Krathwohl

\begin{tabular}{|l|l|}
\hline Categoría & Procesos cognitivos involucrados \\
\hline $\begin{array}{l}\text { Recordar: recuperar conocimiento relevante } \\
\text { desde la memoria de largo plazo. }\end{array}$ & $\begin{array}{l}\text { - Reconocer } \\
\text { - Recordar }\end{array}$ \\
\hline $\begin{array}{l}\text { Entender: determinar el significado de } \\
\text { mensajes instruccionales, incluyendo } \\
\text { comunicación oral, escrita y gráfica. }\end{array}$ & - Interpretar \\
& - Ejemplificar \\
& - Clasificar \\
& - Resumir \\
& - Inferir \\
\hline
\end{tabular}




\begin{tabular}{|l|l|}
\hline & - Comparar \\
& - Explicar \\
\hline $\begin{array}{l}\text { Aplicar: Usar un procedimiento en una } \\
\text { situación determinada. }\end{array}$ & - Ejecutar \\
& - Implementar \\
\hline $\begin{array}{l}\text { Analizar: Dividir un material en sus partes } \\
\text { constituyentes y detectar como las partes se } \\
\text { relacionan unas con otras, y con una } \\
\text { estructura general y un propósito. }\end{array}$ & - Diferenciar \\
$\begin{array}{l}\text { Elarganizar } \\
\text { Evaluar: Juzgar con base en criterios y } \\
\text { estándares. }\end{array}$ & - Revisar \\
\hline $\begin{array}{l}\text { Crear: Articular elementosignar valores y } \\
\text { todo nuevo y coherente, o hacer un producto } \\
\text { original. }\end{array}$ & - Criticar \\
\hline
\end{tabular}

Fuente: Krathwohl (2002, p. 215).

Si bien los planes de estudio de primaria publicados por el Ministerio de Educación Pública de Costa Rica indican los objetivos de aprendizaje que deben abordarse en las aulas, los objetivos específicos, definidos por las personas docentes para abordar cada una de las actividades de aprendizaje realizadas a lo largo del ciclo lectivo, solo pueden identificarse de manera limitada, mediante sistematizaciones localizadas y exhaustivas.

A pesar de esta limitación causada por la naturaleza misma del objeto de investigación, se sugiere que las actividades de aprendizaje mediadas con las computadoras XO y sustentadas en la integración de objetos de aprendizaje, pueden corresponder mayoritariamente a objetivos de aprendizaje vinculados a las categorías y procesos cognitivos de mayor complejidad: aplicar, analizar, evaluar y crear. Esta premisa fue tomada en consideración para categorizar las actividades que realizan las personas docentes y estudiantes, utilizando recursos audiovisuales y multimedia, y para guiar la identificación de cuáles de estas actividades efectivamente pueden considerarse como instancias de aprovechamiento de objetos de aprendizaje. 


\section{Utilización de recursos audiovisuales y multimedia como objetos de aprendizaje en las computadoras XO}

Con el objetivo de identificar tendencias en la construcción de ambientes de aprendizaje que integren el uso de objetos de enseñanza y objetos de aprendizaje en las escuelas del Proyecto Conectándonos, se aplicó una encuesta de 26 preguntas (25 cerradas y una abierta) a las personas docentes de las escuelas incluidas en el proyecto hasta el inicio del 2014. A través de la aplicación de este instrumento se recolectó información sobre las características demográficas del cuerpo docente (sexo, edad, nivel y asignaturas a su cargo), las frecuencias y espacios de utilización de las computadoras XO (frecuencia de utilización general y por asignatura, lugares de utilización dentro y fuera de la escuela, y recursos tecnológicos utilizados en conjunto con las computadoras $\mathrm{XO}$ ) y la Interacción entre estudiantes, docentes y objetos de aprendizaje (categorizadas como actividades de aprendizaje de lectura, modificación y construcción).

La muestra estuvo conformada por personas docentes a cargo de construir ambientes de aprendizaje en las escuelas del Proyecto Conectándonos. De este modo, se excluyeron de la muestra a las personas directoras y administrativas de los centros de enseñanza, que señalaron no tener lecciones a su cargo. Entre octubre de 2014 y enero del 2015 se buscó contactar vía telefónica a las 297 personas incluidas en las bases de datos facilitadas por la Fundación Quirós Tanzi, de las cuales se contactó exitosamente a 186. Las llamadas se realizaron en horas de la tarde y la noche de días escolares, y en las mañanas de fines de semana, con el fin de no interrumpir a las personas docentes en sus jornadas de trabajo.

Es importante mencionar que 30 de las personas contactadas se negaron a realizar la encuesta $\mathrm{O}$ indicaron que no utilizaban las computadoras XO. Estas personas fueron excluidas de los resultados presentados en este apartado. De este modo, se presenta la sistematización de las respuestas de 156 personas docentes. Cabe señalar también que 45 de los números telefónicos de personas incluidas en la lista eran incorrectos o no se encontraban disponibles. El resto de números telefónicos incluidos en la lista corresponden a casos en los que el número es válido, pero no fue posible obtener respuesta por parte de las personas docentes.

Entre las personas docentes que respondieron a la encuesta, $71 \%$ estuvo a cargo de una de las cuatro asignaturas básicas (español, estudios sociales, matemática, ciencias) y 29 \% trabajaba con alguna de las asignaturas especiales (inglés, educación física, educación para el hogar, educación especial, artes plásticas, etc.). La mayoría de las personas 
encuestadas fueron mujeres $(74,4 \%)$ y personas con edades entre los 35 y 50 años $(60,2$ $\%)$.

Tras definir el perfil demográfico y las asignaturas trabajadas por las personas docentes, se recolectó información relacionada con la frecuencia y los espacios de utilización de las computadoras XO. Como se muestra en la tabla No.2, solamente $16 \%$ de las personas docentes dicen utilizar las computadoras $\mathrm{XO}$ todos los días que imparten lecciones. Las proporciones más altas corresponden a quienes las utilizan entre una y cinco veces a la semana. De este modo, es posible inferir que las personas docentes en su mayoría han incorporado la computadora XO a sus dinámicas de clase siguiendo una frecuencia moderada, que permite la alternación de actividades de aprendizaje mediadas por tecnologías digitales, con técnicas didácticas tradicionales, sustentadas en interacciones orales, actividades de lectura y escritura, y utilización de material impreso.

Vale resaltar que casi una quinta parte de las personas que indican utilizar las computadoras señala que lo hace menos de un día a la semana, lo que cual sugiere un desaprovechamiento parcial de los dispositivos en las escuelas del Proyecto.

Tabla No.2

Frecuencia de utilización de computadoras XO

\begin{tabular}{|l|l|}
\hline Frecuencia de utilización & Porcentaje \\
\hline Todos los días & $16 \%$ \\
\hline De 3 a 5 días por semana & $26,9 \%$ \\
\hline De 2 días a 1 día por semana & $39,7 \%$ \\
\hline Menos de 1 día por semana & $17,3 \%$ \\
\hline
\end{tabular}

Fuente: Elaboración propia del autor (2016).

Adicionalmente, con el fin de dilucidar los objetos de estudio con los que da un mayor aprovechamiento de los dispositivos, se consultó a las personas docentes sobre las asignaturas en las que realizan un uso más extensivo de las computadoras XO. Las asignaturas que presentan una utilización más frecuente corresponden a español $(26,9 \%)$, estudios sociales $(25,6 \%)$ y ciencias $(24,3 \%)$. Por su parte, matemáticas presenta una frecuencia de utilización considerablemente menor que las otras tres materias básicas $(18,5$ $\%)$, lo cual podría deberse a que las personas docentes se encuentran con un mayor grado de dificultad para adaptar los objetos de estudio de matemática a actividades de aprendizaje mediadas con las computadoras XO.

De la misma manera, se manifiesta una utilización particularmente limitada en la asignatura de inglés como segundo idioma y en el resto de asignaturas especiales. Estos 
resultados llaman la atención sobre la necesidad de emprender acciones que favorezcan la utilización de las computadoras XO en las asignaturas de matemática e inglés.

Tabla No.3

Frecuencia de utilización según asignatura

\begin{tabular}{|l|l|}
\hline Asignatura & Porcentaje \\
\hline Español & $26,9 \%$ \\
\hline Sociales & $25,6 \%$ \\
\hline Matemática & $18,5 \%$ \\
\hline Ciencias & $24,3 \%$ \\
\hline Inglés & $11,5 \%$ \\
\hline Otras especiales & $16,6 \%$ \\
\hline
\end{tabular}

Fuente: Elaboración propia del autor (2016).

Si bien las actividades de aprendizaje que incorporan el aprovechamiento de objetos de aprendizaje pueden realizarse dentro del espacio físico del aula, al que tradicionalmente se circunscriben las dinámicas de aprendizaje de las escuelas primarias, la "colonización" de otros espacios físicos de los centros educativos, tales como jardines, bibliotecas y zonas deportivas, posibilitan a las y los estudiantes interactuar de nuevas maneras con sus dispositivos digitales, con su entorno, y con sus pares.

De este modo, una variedad en los espacios de utilización de las computadoras XO no solamente sugiere la posibilidad de que ya algunas personas docentes sacan a los niños y niñas de las aulas para realizar actividades que aprovechan objetos de aprendizaje, sino que también facilita las condiciones para introducir dichos objetos a las prácticas de enseñanza de la comunidad docente, a través de mecanismos que pueden incluir la producción y distribución de recursos audiovisuales y multimedia.

Tomando en consideración estas reflexiones, se consultó a las personas docentes sobre los espacios en los que utilizan las computadoras XO. A pesar de que la utilización por parte de las personas docentes se concentra adentro de las aulas, en la tabla No.4 es posible reconocer que la utilización en otros espacios también relativamente frecuente, entre los que sobresalen los pasillos y los jardines o patios de las escuelas. Es importante resaltar que en la aplicación de la encuesta se identificó el año en que cada escuela se había incorporado al Proyecto Conectándonos, y fue posible observar que la proporción de las personas que dicen aprovechar los distintos espacios presenta algunas variaciones dependiendo de dicho año.

Estas variaciones pueden responder a factores como la maduración de los procesos del Proyecto y las condiciones particulares de las escuelas incorporadas en cada año. Por 
ejemplo, las personas docentes de escuelas que ingresaron al proyecto en el 2013 muestran un mayor aprovechamiento de los distintos espacios del centro educativo. Esta tendencia puede responder a una utilización más asertiva de los dispositivos, facilitada por la consolidación de los procesos de formación a las personas docentes durante dicho año. Del mismo modo, se puede apreciar que en las escuelas que ingresaron al Proyecto en el 2013 y 2014, las cuales son predominantemente rurales, hay un uso más extensivo de los patios y jardines.

Tabla No.4

Espacios de utilización de computadoras XO

\begin{tabular}{|l|l|l|l|l|}
\hline Espacio de utilización & $\mathbf{2 0 1 2}$ & $\mathbf{2 0 1 3}$ & $\mathbf{2 0 1 4}$ & Promedio \\
\hline Adentro del aula & $\begin{array}{l}89 \% \\
\text { En los pasillos de la escuela }\end{array}$ & $\begin{array}{l}95,8 \\
\%\end{array}$ & $\begin{array}{l}89,2 \\
\%\end{array}$ & $923 \%$ \\
\hline En el patio/ jardín de la escuela & $\begin{array}{l}30,1 \\
\%\end{array}$ & $\begin{array}{l}17,8 \\
\%\end{array}$ & $23,7 \%$ \\
\hline En las zonas deportivas de la escuela & $\begin{array}{l}25,4 \\
\%\end{array}$ & $\begin{array}{l}31,4 \\
\%\end{array}$ & $\begin{array}{l}\text { 46,4 } \\
\%\end{array}$ & $32 \%$ \\
\hline En la biblioteca de la escuela & 10,9 & $\begin{array}{l}17,7 \\
\%\end{array}$ & $3 \%$ & $11,5 \%$ \\
\hline Afuera de la escuela & $1,8 \%$ & $0 \%$ & $0 \%$ & $0,6 \%$ \\
\hline
\end{tabular}

Fuente: Elaboración propia del autor (2016).

La caracterización y la indagación de la forma en que las y los estudiantes interaccionan con los recursos, puede brindar pistas sobre si estos recursos están siendo utilizados como objetos de aprendizaje, o están siendo limitados a su dimensión de objetos de enseñanza. De este modo, se establecieron categorías para agrupar las actividades de aprendizaje en las que la persona docente incorpora recursos audiovisuales o multimedia a la mediación con las computadoras XO: actividades de lectura, de modificación y de creación.

El establecimiento de estas categorías se fundamentó en el concepto de objeto de aprendizaje propuesto en el apartado anterior, considerando los indicadores de podrían ser observados en actividades que incluyan o excluyan este tipo de objetos. Cabe mencionar que se recurrió a esta categorización para facilitar la recolección de información sobre la manera en que las personas docentes utilizan distintos recursos en convergencia con las computadoras $\mathrm{XO}$, sin tener que brindarles, durante la aplicación del instrumento, una definición de objeto de aprendizaje que pudiera generar sesgos previos en la manera en que comprende y describe sus prácticas de mediación en la construcción del aprendizaje. 
Estas categorías guardan relación con las presentadas en la revisión de la taxonomía de Bloom realizada por Krathwohl. La categoría de las actividades de lectura responde a los procesos cognitivos vinculados con las categorías de "recordar" y "entender", la de actividades de modificación se relaciona con las de "aplicar" y "analizar", y la de actividades de creación con la de "crear". Siguiendo esta correspondencia, se plantea que los objetivos de aprendizaje de las actividades coincidentes con las categorías propuestas para esta investigación, pueden corresponder a los infinitivos verbales propuestos por la taxonomía de Krathwohl para enunciar los distintos procesos cognitivos.

Con el instrumento de encuesta se recolectó información sobre distintos recursos audiovisuales y multimedia, agrupados a partir de afinidades tanto en los lenguajes que utilizan y como en el tipo de información que transmiten a través de estos lenguajes (fotografías e ilustraciones, mapas y esquemas, videos educativos, música, videojuegos). Asimismo, se consultó si las actividades de aprendizaje mediadas con los recursos habían sido realizadas individual o grupalmente por parte de las y los estudiantes.

Por una parte, la información ofrecida por las personas docentes indica que en la mayoría de actividades de aprendizaje, el aprovechamiento de los recursos audiovisuales se limitó una utilización pasiva, característica del acercamiento a los recursos como objetos de enseñanza. Estos acercamientos se identifican como actividades de lectura, en las cuales el estudiantado participó mayoritariamente de manera individual. Vale resaltar que la actividad de aprendizaje realizada por la mayor proporción de docentes corresponde a la "lectura" de fotografías e ilustraciones $(68,5 \%)$.

Por otra parte, las actividades de modificación, en las que el estudiantado manipula la forma o el contenido de un recurso audiovisual o multimedia facilitado por la persona docente, son las menos comunes. Esta tendencia denota un vacío provocado posiblemente por la dificultad de las personas docentes para diseñar y elaborar recursos sensibles a ser modificados en el contexto de una actividad de aprendizaje. Mientras que las actividades de modificación en las que se utilizan fotografías e ilustraciones son las más referenciadas por las personas docentes (41\%), las que incorporan los videos educativos y los videojuegos son las menos comunes.

Finalmente, las actividades de construcción se proponen como aquellas en las que se conciben los recursos audiovisuales y multimedia como objetos de aprendizaje. Si bien estas actividades se realizan también de manera limitada, presentan números relativamente altos en el caso de la creación individual de fotografías e ilustraciones (64,1\%), y esquemas y mapas $(49,9 \%)$, lo cual puede responder a las facilidades de hardware (cámara digital 
incorporada) y software (aplicaciones para la elaboración de mapas mentales) brindadas por las computadoras XO.

Tabla No.5

Realización de actividades de lectura, modificación y construcción

\begin{tabular}{|l|l|l|l|l|}
\hline Recurso & Tipo de Actividad & Individualmente & Grupalmente & Sin uso \\
\hline \multirow{3}{*}{$\begin{array}{l}\text { Fotografías, } \\
\text { Ilustraciones }\end{array}$} & Lectura & $68,5 \%$ & $62,1 \%$ & $14,1 \%$ \\
\cline { 2 - 5 } & Modificación & $41,0 \%$ & $20,5 \%$ & $53,2 \%$ \\
\cline { 2 - 5 } & Construcción & $64,1 \%$ & $32 \%$ & $24,9 \%$ \\
\hline \multirow{3}{*}{$\begin{array}{l}\text { esquemas, } \\
\text { Videos }\end{array}$} & Lectura & $40,3 \%$ & $32,6 \%$ & $40,3 \%$ \\
\cline { 2 - 5 } & Modificación & $30,1 \%$ & $14,1 \%$ & $61,5 \%$ \\
\cline { 2 - 5 } & Construcción & $49,9 \%$ & $26,9 \%$ & $39,1 \%$ \\
\hline \multirow{4}{*}{ Música } & Lectura & $51,2 \%$ & $60,2 \%$ & $12,8 \%$ \\
\cline { 2 - 5 } & Modificación & $11,5 \%$ & $7,0 \%$ & $83,3 \%$ \\
\cline { 2 - 5 } & Construcción & $29,4 \%$ & $24,9 \%$ & $54,4 \%$ \\
\cline { 2 - 5 } & Lectura & $39,7 \%$ & $39,7 \%$ & $33,3 \%$ \\
\cline { 2 - 5 } & Modificación & $7,0 \%$ & $10,8 \%$ & $87,1 \%$ \\
\cline { 2 - 5 } & Construcción & $10,8 \%$ & $14,7 \%$ & $87,1 \%$ \\
\cline { 2 - 5 } & Lectura & $32,6 \%$ & $2,5 \%$ & $93,6 \%$ \\
\cline { 2 - 5 } & Modificación & $4,4 \%$ & $4,4 \%$ & $91,6 \%$ \\
\cline { 2 - 5 } & Construcción & $4,4 \%$ & $2016)$ & \\
\hline
\end{tabular}

Fuente: Elaboración propia del autor (2016).

Mediante la indagación de las prácticas de mediación de las personas docentes, y su relación con el uso de objetos de aprendizaje, se identificaron las tareas pendientes en el abordaje metodológico para el aprovechamiento de los recursos audiovisuales y multimedia como objetos de aprendizaje, en el contexto de un modelo de acceso uno a uno.

Los hallazgos obtenidos mediante la aplicación del instrumento de encuesta fueron sistematizados en el diseño y elaboración de recursos audiovisuales y multimedia para que sean aprovechados en actividades que incorporen el uso de objetos de aprendizaje, en las escuelas del Proyecto Conectándonos, y que a la vez aborden objetivos y contenidos de los Planes de Estudio del Ministerio de Educación Pública.

Cabe mencionar que la encuesta también incluyó preguntas sobre la manera en que las personas docentes perciben la manifestación entre sus estudiantes de los indicadores de la competencia de colaboración, incluida entre el marco de competencias ATC21S. Los resultados correspondientes a este punto fueron presentados en una publicación anterior. (Mena, 2015) 


\section{Diseño y producción de recursos audiovisuales y multimedia como objetos de aprendizaje}

La carrera de Ciencias de la Comunicación Colectiva de la Universidad de Costa Rica incluye en el plan de estudios de sus cinco carreras (Periodismo, Comunicación Audiovisual y Multimedia, Relaciones Públicas, Publicidad y Comunicación Social) dos cursos denominados Módulo Integrador I y Módulo Integrador II, en los cuales se articulan las tres áreas sustantivas del quehacer universitario: la docencia, la investigación y la acción social. En estos cursos las personas estudiantes colaboran con una persona docente en el desarrollo de proyectos de investigación que buscan generar un impacto positivo en espacios comunitarios e institucionales, y al mismo tiempo convertirse en una plataforma para el aprendizaje de habilidades en el diseño e implementación de investigaciones.

Durante el primer semestre del 2015, comprendido entre la primera semana de marzo y la primera semana de julio, los docentes Aarón Mena y Rodrigo Muñoz-González coordinaron el diseño y producción de dos recursos con elementos audiovisuales y multimedia dirigidos a las a escuelas primarias vinculadas al Proyecto Conectándonos en el marco del curso Módulo Integrador I. En estas tareas participaron 14 personas estudiantes, provenientes de las distintas carreras de la Escuela de Ciencias de la Comunicación Colectiva: Andrea Alfaro, Melany Alvarado, Andrey Barrantes, Noelia Elizondo, Tatiana Espinoza, Julián Godoy, Laura Gordillo, Paola Guzmán, Anthony Martén, Irina Méndez, Valeria Miranda, Alejandro Pérez, Jessie Ramos y Virginia Soley. Adicionalmente, el estudiante Julián Berkovics participó como fotógrafo y asistente en las tareas de producción.

El diseño y producción de los recursos educativos se sustentó en tres fuentes de información fundamentales: los planes de estudio del Ministerio de Educación Pública para primaria, la información recopilada por la Fundación Quirós Tanzi sobre el Proyecto Conectándonos, y los resultados de la aplicación de instrumentos de recolección de datos por las personas docentes y estudiantes del curso de Módulo Integrador I.

Como parte de las primeras tareas del curso, las personas estudiantes realizaron de manera conjunta y en coordinación con las personas docentes, el diseño y aplicación de instrumentos de recolección de datos orientadas a dilucidar las características de las prácticas de utilización de recursos audiovisuales y multimedia en una de las escuelas del Proyecto Conectándonos. Para este fin se seleccionó a la Escuela Barrio del Socorro, ubicada en el cantón de Santo Domingo, en la provincia de Heredia. La figura No. 1 muestra a las personas estudiantes participando en las actividades de investigación. Vale mencionar 
que la Escuela Barrio del Socorro ingresó al Proyecto Conectándonos en el 2014, con 11 docentes y 46 estudiantes.

Figura No.1

Estudiantes del Módulo Integrador I en la Escuela Barrio del Socorro

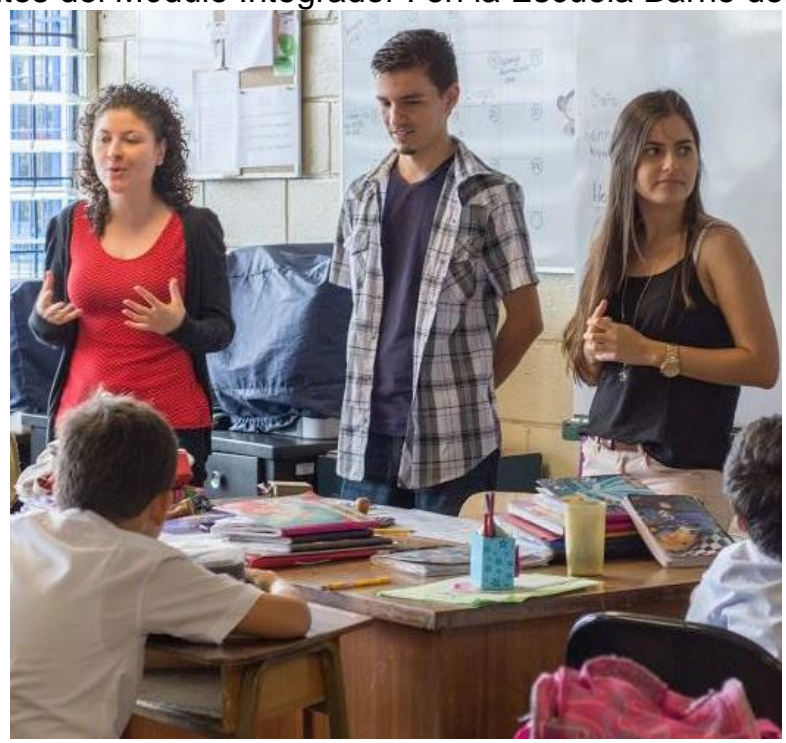

Fotografía de Julián Berkovics (2015).

El primer instrumento de recolección de datos correspondió a una guía de observación no participante, el cual fue aplicado en una visita a la Escuela Barrio del Socorro, el 17 de abril del 2015. En esta visita las personas estudiantes del curso se dividieron en grupos de trabajo y realizaron tareas de observación durante un bloque de lecciones, constituido por dos lecciones de 40 minutos cada una. Al terminar la primera lección, los grupos de trabajo se desplazaron al aula correspondiente a otro grado, con lo cual cada uno de los grupos tuvo la oportunidad de observar las dinámicas de dos grados distintos.

El instrumento de observación no participante recopiló información sobre nueve variables: el estado de la infraestructura de la escuela, la distribución del mobiliario dentro de las aulas, la ubicación de las computadoras XO dentro del aula, la interacción de los niños y niñas con sus pares cuando utilizan la $X O$, la interacción de los niños y niñas con las computadoras $\mathrm{XO}$, la interacción de la persona docente con las computadoras $\mathrm{XO}$, la interacción de la persona docente con los niños y niñas, la utilización conjunta de recursos audiovisuales y multimedia con las computadoras $\mathrm{XO}$, y la utilización de las XO fuera de las aulas.

La información recolectada fue presentada y discutida, para luego construir de manera conjunta una lista de conclusiones que servirían de guía en el diseño de los recursos audiovisuales y multimedia, y actividades de aprendizaje complementarias. En el recuadro 
No.1 se detallan se detallan los principales hallazgos del proceso de observación participante. 
Recuadro No.1

Resumen de hallazgos de observación no participante

1. Se determinó que las aulas eran compartidas por distintos grados, lo que podría dificultar la realización de actividades de aprendizaje dentro de las aulas. Se tomó la decisión de diseñar actividades que también pudieran ser desarrolladas en el patio y en los pasillos de la escuela.

2. Se comprobó que la conexión a internet disponible en la escuela permitía la utilización individual y simultánea de recursos multimedia por parte de los niños y las niñas.

3. Se observó que la concentración de pupitres dificulta el movimiento de los niños y las niñas dentro de las aulas, y se decidió que las actividades de aprendizaje ligadas a los recursos audiovisuales y multimedia fueran diseñadas para desarrollarse trabajando en el pupitre o en los espacios abiertos de la escuela.

4. Se evidenció que los niños y las niñas son reticentes a compartir una computadora con uno de sus pares, y que en los casos en que deben hacerlo, se manifiesta una asimetría en el aprovechamiento de los recursos y actividades de aprendizaje debido al acaparamiento de la herramienta por parte de su dueño o dueña. Así, se identificó la necesidad de diseñar recursos y actividades que pudieran desarrollarse de manera conjunta y colaborativa y generar espacios de diálogo y socialización, pero que también permitieran a cada niño y niña realizar tareas de modificación y creación en su propia computadora XO.

5. Se corroboró que los niños y las niñas dominan el sistema operativo y muchas de las aplicaciones de las computadoras $\mathrm{XO}$, lo que permite el planteamiento de actividades de aprendizaje que aprovechen dichas aplicaciones.

6. Se observó que las personas docentes generalmente no logran revisar el trabajo que realizan todas las personas estudiantes en sus computadoras $\mathrm{XO}$, lo cual hace necesario incluir en los recursos información detallada y oportuna, que le permita a los niños y las niñas consultar por su propia cuenta las acciones que conforman las actividades de aprendizaje mediadas con recursos audiovisuales y multimedia.

Fuente: Elaboración propia del autor (2016).

En una segunda visita a la Escuela Barrio del Socorro realizada el 29 de mayo del 2015, se llevó a cabo una segunda aplicación de instrumentos de recolección de datos. En esta ocasión, las personas estudiantes del curso se dividieron en cinco grupos de trabajo, y durante un bloque de lecciones interactuaron con los niños y las niñas de distintos grados a través de actividades lúdicas. Cada grupo de trabajo interactuó con entre 7 y 16 niños y niñas, realizando las siguientes cinco actividades, cada una de las cuales se extendió por alrededor de diez minutos: 
1. Actividad motivadora: Se presentaron las personas participantes y se introdujo el tono lúdico y dinámico de las actividades, y consistió en juegos con balones, aros de gimnasia y otros materiales.

2. Actividad de intercambio de gustos: Los niños y niñas compartieron detalles sobre sus prácticas de consumo mediático, mientras jugaban con un balón.

3. Técnica proyectiva I: Los niños y niñas representaron mediante dibujos espacios que consideran cómodos y amenos.

4. Técnica proyectiva II: Los niños y niñas expresaron sus sentimientos e ideas sobre las actividades de aprendizaje mediadas con nuevas digitales, mediante la participación en juegos de roles.

5. "Arma tu computadora": En esta actividad los niños y niñas describieron las características de lo que consideran una computadora ideal, dando pistas sobre cuáles de las aplicaciones incluidas en las computadoras XO capturan su interés en mayor grado.

Estas actividades permitieron recolectar información sobre tres variables fundamentales: la utilización que los niños y niñas hacen de las computadoras XO fuera de la escuela, en su hogar o en otros espacios de la comunidad, coincidencias en los productos mediáticos (programas de televisión, películas, cuentos y videojuegos) que los niños y niñas incluyen dentro de sus gustos personales, y la percepción de los niños y niñas hacia las actividades de aprendizaje mediadas con tecnologías digitales. Tras sistematizar la información brindada por los niños y niñas, se hallaron las siguientes tendencias:

- La mayoría de los niños y niñas afirmaron no utilizar las computadoras XO en sus hogares, debido a que tienen acceso a computadoras más potentes, teléfonos móviles y otros dispositivos que aprovechan para jugar videojuegos, acceder a redes sociales, y otras actividades de entretenimiento. De este modo, se puede inferir que los niños y niñas ven a la computadora XO como una herramienta exclusiva para el trabajo en la escuela, por lo que los recursos utilizados en clase no competirían directamente con los productos mediáticos consumidos en el hogar.

- Los niños y niñas guardan un ambiente de participación y respeto; sin embargo al trabajar en equipo se acercan a personas de su mismo género.

- Si bien los niños y niñas expresan gustos variados en su consumo de productos mediáticos, coinciden en el interés por dibujos animados cuyos personajes son seres fantásticos. 
La información recopilada mediante estos instrumentos fue integrada al diseño de los elementos formales (diseño de personajes, diseño gráfico, sonorización, etc.) de los recursos audiovisuales y multimedia, y tomada en consideración en el diseño de las actividades de aprendizaje complementarias. Para realizar estas tareas las personas estudiantes del curso Módulo Integrador se dividieron en dos grupos de trabajo, cada uno conformado por 7 personas.

Asimismo, tomando en consideración la información ofrecida por la encuesta aplicada a las personas docentes del proyecto, se decidió elaborar recursos que atendieran contenidos de las asignaturas de matemáticas e inglés, y que permitieran la realización de tareas de creación y modificación. Además se identificaron las competencias a ATC21S cuyo desarrollo transversal podría ser abordado mediante la utilización de los recursos diseñados.

El primer recurso abordó contenidos del plan de estudios para matemática, y el segundo abordó contenidos de inglés. A continuación se describen los resultados de los procesos de diseño y producción de estos recursos.

\section{Geofotografía}

El primer recurso se denomina "Geofotografía", y busca brindarles a los niños y las niñas del Proyecto Conectándonos la oportunidad de acercarse a conceptos de geometría mientas exploran y representan el mundo a su alrededor. De este modo, se pretende crear un recurso que promueva entre los niños y niñas una visión de la matemática como una herramienta para la comprensión y descripción de objetos familiares y tangibles.

Figura No.2

Logotipo de recurso Geofotografía

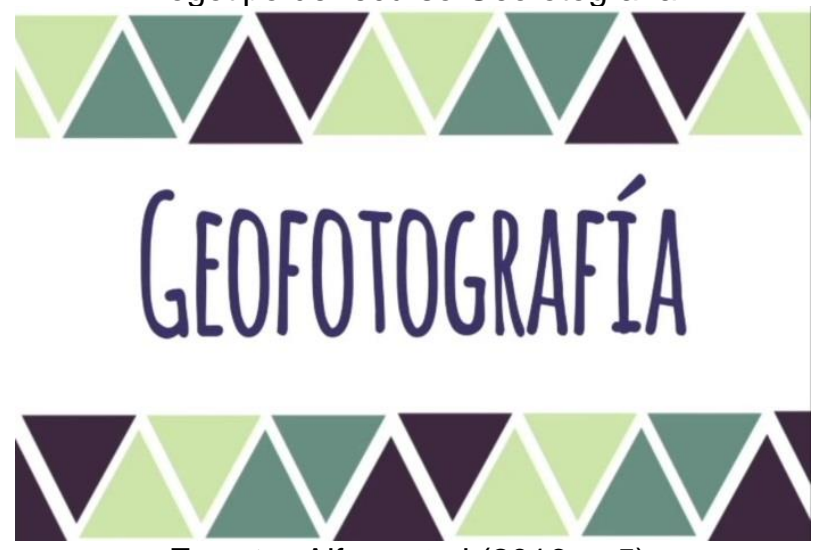

Fuente: Alfaro et al (2016, p.5). 


\subsection{Contenidos abordados}

Geofotografía aborda contenidos del plan de estudios de la asignatura de matemática para cuarto grado (segundo ciclo), específicamente del módulo de geometría. Los contenidos seleccionados para ser representados en el recurso corresponden al cuarto grado, y se centran en conceptos relacionados con los triángulos (lado, vértice, ángulo, base, altura, clasificación de triángulos según la medida de sus lados y sus ángulos, etc.) y los cuadriláteros (lado, vértice, ángulo, base, altura. Diagonal, paralelogramos, rectángulo, rombo, romboide, cuadrado, no paralelogramos, trapecio, trapezoide, etc.).

\subsection{Componentes del recurso}

Los recursos diseñados en el marco del curso de Módulo Integrador I integran productos fundamentados en distintos lenguajes y formatos, que se complementan mutuamente para facilitar el desarrollo de una actividad de aprendizaje en la que los niños y las niñas puedan entrar en contacto con objetos de aprendizaje.

Geofotografía está compuesto por un video de instrucciones dirigido principalmente a las personas docentes, en el cual se detalla el rol de docente y estudiantes en la realización de una actividad de aprendizaje que aprovecha dos aplicaciones disponibles en las computadoras XO, denominadas "Grabar" y "Geogebra". "Grabar" permite tomar fotografías y videos utilizando la cámara incorporada en la computadora. "Geogebra" permite construir figuras geométricas trazando y uniendo líneas, ofreciendo información sobre la medida de los lados y ángulos de dichas figuras.

Cabe mencionar que el video fue grabado en las instalaciones de la Escuela Barrio del Socorro, con la participación de niños y niñas de cuarto grado.

Figura No.3

Video tutorial de instrucciones

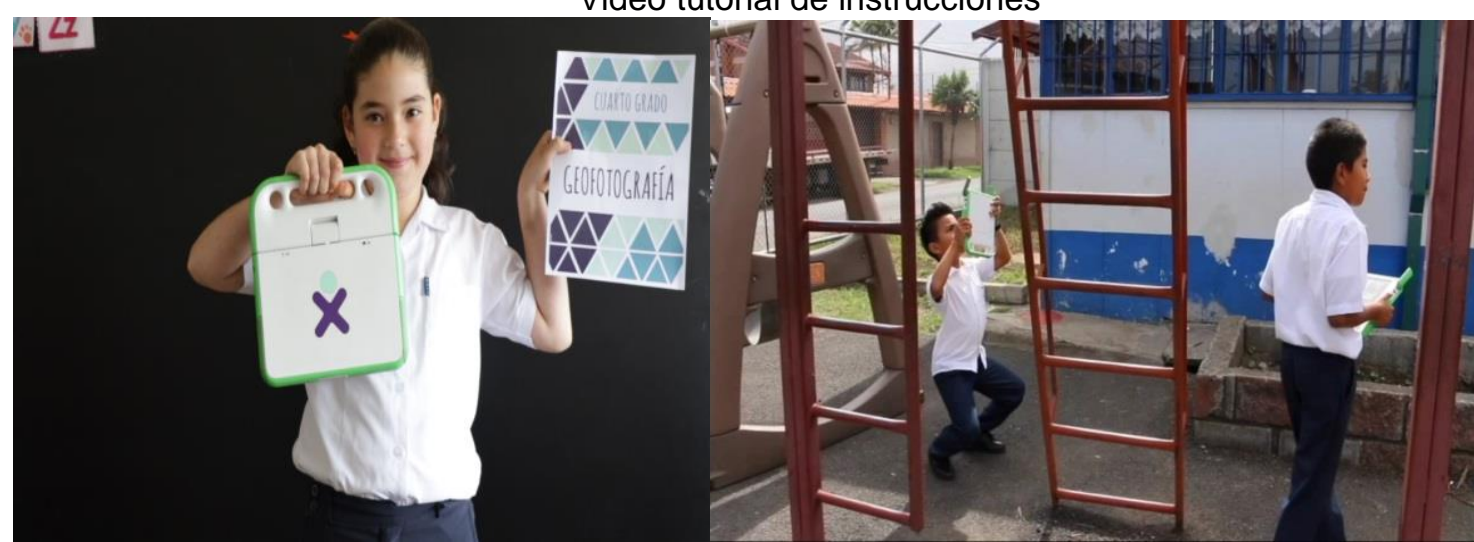

Fuente: Alfaro et al (2015, p.6). 
El recurso incluye también dos manuales, uno para uso de la persona docente y otro para uso de las personas estudiantes. Por una parte, el primero le brinda instrucciones detalladas a la persona docente sobre la dinámica y el sustento metodológico y teórico $v$ de la actividad de aprendizaje. Por otra parte, el manual dirigido a estudiantes sirve de cuaderno de campo en el desarrollo de la actividad de aprendizaje. Estos manuales se distribuyen en formato PDF para que puedan ser impresos por la persona docente, y distribuidos a los niños y niñas.

\subsection{Actividad de aprendizaje complementaria}

La actividad de aprendizaje complementaria está diseñada para que los niños y las niñas utilicen los componentes del recurso Geofotografía como objetos de aprendizaje. De este modo, se busca que el recurso les ayude a visualizar su apropiación de los conceptos de geometría, mediante la observación guiada de su entorno y la creación de sus propios archivos digitales. Los pasos de la actividad de aprendizaje se describen en el recuadro No.2.

Recuadro No.2

Pasos de actividad de aprendizaje complementaria para Geofografía 1. La persona docente explica los pasos de la actividad a las niños y niñas, para luego distribuirles los manuales impresos. Es importante que para realizar la actividad los niños y las niñas puedan movilizarse libremente por las instalaciones de la escuela.

2. Los niños y las niñas utilizando la aplicación "Grabar" de la computadora XO toman fotografías de la infraestructura y mobiliario de la escuela, en busca de las figuras geométricas estudiadas. El manual incluye los nombres, descripciones e ilustraciones de las figuras que deben buscar.

3. Los niños y las niñas guardan las fotografías en el portapapeles de la computadora XO y las suben a la aplicación "Geogebra".

4. Los niños y las niñas trazan las figuras geométricas sobre las fotografías subidas, y las clasifican según sus ángulos y lados.

5. Los niños y las niñas comparten sus hallazgos con sus pares, bajo la guía de la persona docente.

Fuente: Alfaro et al (2015, p.7).

Es importante mencionar que la actividad fue completada en la Escuela Barrio del Socorro durante un bloque de dos lecciones, por lo que se sugiere que en las escuelas del Proyecto Conectándonos pueda ser replicada en un periodo de tiempo similar. 


\subsection{Competencia ATC21S abordada}

Se espera que la utilización de los recursos de Geofotografía facilite a las personas docentes la realización de una actividad de aprendizaje en la que los niños y niñas puedan tener un acercamiento transversal al desarrollo de la competencia "Literacidad en tecnologías de la información y la comunicación", ubicada en la categoría de "Herramientas para trabajar" de las compentencias ATC21S.

Es importante mencionar que cada competencia ATC21S cuenta con una serie de indicadores organizados en las dimensiones de conocimientos, habilidades y actitudes, valores y éticas. Asimismo, dentro de cada dimensión se proponen acciones generales que agrupan a los indicadores.

Geofotografía posibilita particularmente la visualización de dos indicadores. El primero corresponde a la dimensión de conocimientos, y hace referencia a la creación de productos mediáticos: "Comprende y sabe cómo utilizar las herramientas, características y convenciones más apropiadas para la creación mediática" (Griffin et al., 2012, p. 52). El segundo indicador corresponde a la dimensión de habilidades y hace referencia a la aplicación efectiva de la tecnología: "Usa la tecnología como una herramienta para investigar, organizar, evaluar y comunicar información". (Griffin et al., 2012, p. 52)

\section{Name it}

El segundo recurso se denomina "Name it" y articula video tutoriales y juegos de memoria para apoyar a los niños y niñas en el aprendizaje de vocabulario para la asignatura de inglés.

Figura No. 4

Logotipo de recurso Name it

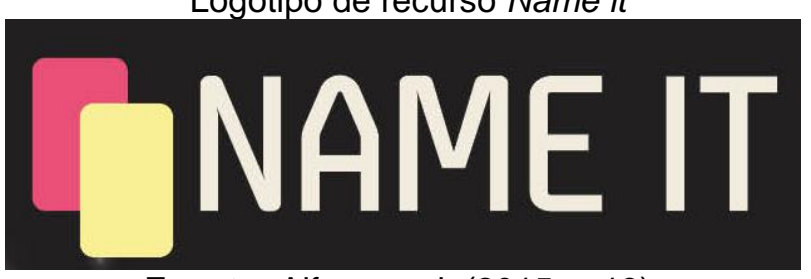

Fuente: Alfaro et al, (2015, p.13). 


\subsection{Contenidos abordados}

Name it aborda contenidos correspondientes al plan de estudios de la asignatura de inglés para primer grado, y se concentra en la unidad denominada "My Interests". En ésta se incluye vocabulario relacionado con alimentos (frutas, vegetales, carnes, postres y bebidas), mascotas, cuentos, pasatiempos, colores, tamaños, formas y números. Con la selección de estos contenidos se busca ofrecer a las y los estudiantes un recurso que les permita practicar la escucha, pronunciación y lectoescritura de los elementos de léxico con los inician su aprendizaje de la lengua inglesa.

\subsection{Componentes del recurso}

Name it cuenta con cuatro componentes complementarios, entre los que se incluyen un manual para las personas docentes, un video de presentación de vocabulario, un video de instrucciones dirigido a los niños y las niñas, y dos juegos de memoria para ser utilizados en las computadoras XO.

El manual para las personas docentes se distribuye en PDF y describe los objetivos y metodología de Name it. Asimismo, explica detalladamente cómo guiar a los niños y las niñas en la utilización de los distintos recursos, y en la creación de sus propios juegos de memoria.

Figura No. 5

Manual para docentes de Name it
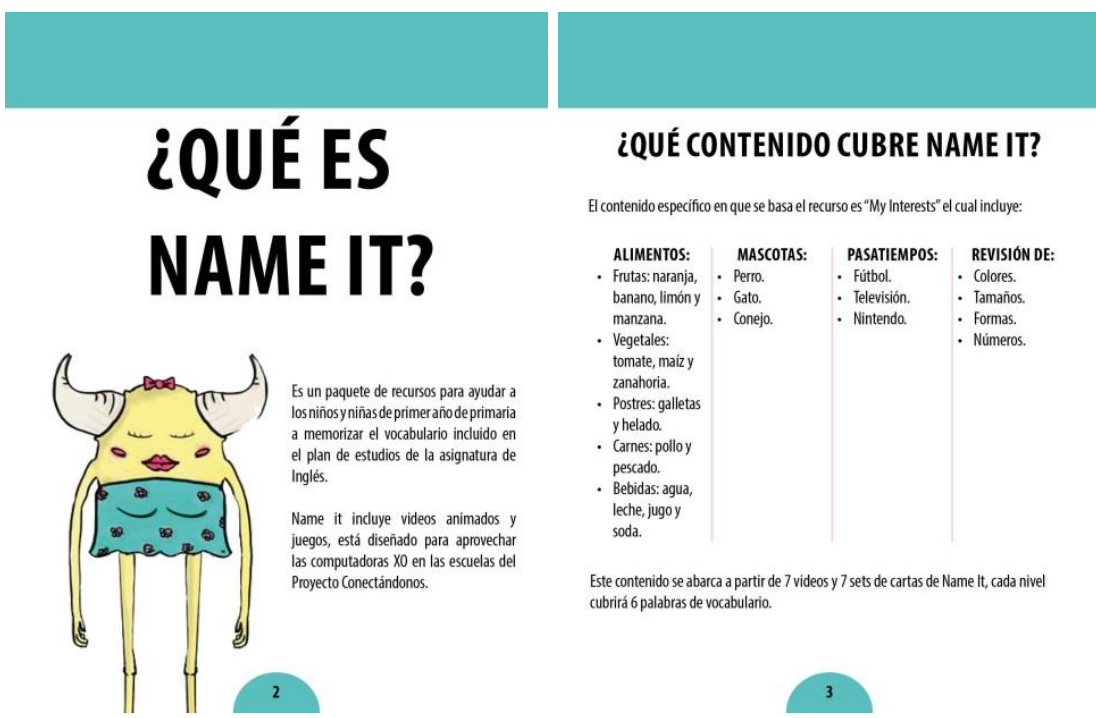

\section{¿QUÉ CONTENIDO CUBRE NAME IT?}

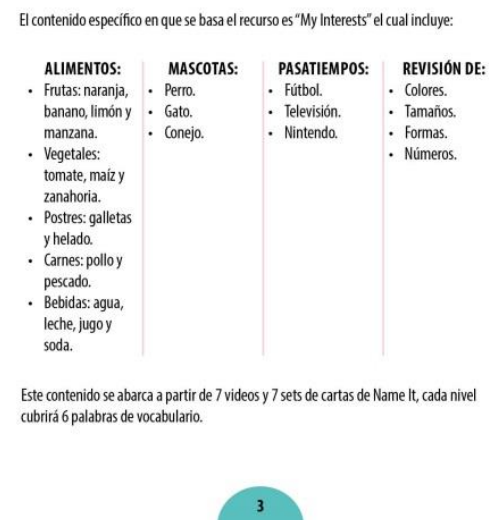

Fuente: Alfaro et al (2015, p.14). 
El video de vocabulario presenta la interacción Xandi y Odric, dos personajes animados que protagonizan pequeñas historias, en las que utilizan el vocabulario de unidad de My Interests, tanto en castellano como en inglés. Por una parte, este video brinda a los niños y las niñas la oportunidad de escuchar la pronunciación de las palabras estudiadas, mientras son utilizadas en oraciones prácticas y sencillas.

Por otra parte, en el video de instrucciones los dos personajes explican a los niños y las niñas cómo abrir los juegos de memoria incluidos en el recurso Name it, y cómo crear después sus propios juegos de memoria.

Vale mencionar que los personajes representan a dos extraterrestres, uno femenino y uno masculino, que llegan a la tierra para experimentar las mismas emociones que experimentan los niños y las niñas. Tomando en consideración la información recolectada mediante las visitas a la Escuela Barrio del Socorro, los personajes fueron diseñados para tener la apariencia de monstruos amables, que emularan la identidad de gráfica de algunas de las series animadas mencionadas por los niños y las niñas como sus favoritas.

Figura No.6

Video de vocabulario de Name it
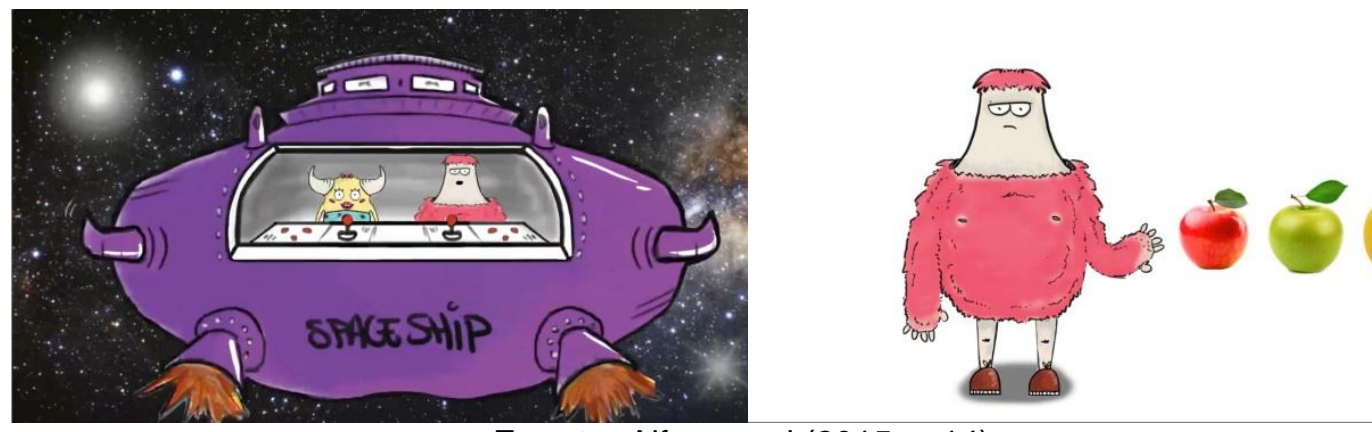

Fuente: Alfaro et al (2015, p.14).

\subsection{Actividad de aprendizaje complementaria}

En la actividad de aprendizaje complementaria, los recursos de Name it se articulan para facilitar una práctica en la que los niños y las niñas pueden asociar el significado, la escritura y la pronunciación de las palabras del vocabulario de la unidad My interests. Al igual que Geofotografía, Name it busca convertirse en un objeto de aprendizaje mediante el cual los niños y las niñas del Proyecto Conectándonos puedan acercarse de una manera lúdica a los contenidos de estudio, para luego crear y socializar sus propios recursos. En el recuadro No.3 se describen los pasos recomendados para el desarrollo de la actividad de aprendizaje complementaria. 
Recuadro No.3

Pasos de actividad de aprendizaje complementaria para Name it

1. Tras haber leído el manual, la persona docente introduce el recurso a los niños y las niñas y habilita el espacio y el tiempo para el visionado del video de vocabulario y el video de instrucciones. Este visionado puede ser gestionado por la persona docente mediante el uso de un proyector, o puede ser realizado de manera individual por los niños y las niñas en sus computadoras XO.

2. Los niños y las niñas, con la guía de la persona docente, descargan los juegos de memoria y los guardan en el diario de su computadora XO. Los juegos corresponden a archivos de la aplicación "Memorizar", la cual permite insertar imágenes y sonidos a tarjetas que pueden ser volteadas y emparejadas en juego de memoria. Al cliquear sobre cada tarjeta, esta se voltea mostrando una ilustración de una de las palabras del vocabulario y su respectiva pronunciación. Se recomienda que los juegos sean realizados en parejas, de manera que los niños y las niñas puedan practicar la pronunciación de las palabras con sus pares.

3. Una vez comprendida la dinámica de los juegos de memoria, utilizando la aplicación "navegar" los niños y las niñas recolectan imágenes relacionados con el vocabulario estudiado, y usando la aplicación "grabar", se graban a sí mismos pronunciando las palabras. Aprovechando las imágenes y las grabaciones de voz, los niños y las niñas crean sus propios juegos de memoria.

4. Utilizando la red mesh, que permite a las XO conectarse entre sí, los niños y las niñas comparten sus nuevos juegos de memoria, y los exploran bajo la guía de la persona docente.

Fuente: Alfaro et al 2015 (p.15-16).

Cabe mencionar que el desarrollo de la actividad de aprendizaje contempla la utilización minuciosa de los tres componentes de Name it (los dos videos y los juegos de memoria), y la creación de recursos propios por parte de los niños y las niñas. Por este motivo, se sugiere que las personas docentes programen el visionado de los videos y la utilización de los juegos de memoria para una sesión de trabajo en un bloque de dos lecciones, y que en una sesión ulterior, facilite el espacio para que creen sus propios juegos de memoria. El valor de esta actividad es que puede replicarse a lo largo del año lectivo, para abordar nuevos contenidos de la asignatura de inglés.

Finalmente, es importante señalar que los recursos de Name it y Geofotografía estarían puestos a disposición de las escuelas del Proyecto Conectándonos a través del "Educatico", el repositorio electrónico del Ministerio de Educación Pública. Tras obtener los consentimientos informados correspondientes, los videos serían incluidos mediante enlaces a la red social Youtube, y los manuales en formato PDF estarían disponibles para su 
descarga directa, al igual que los juegos de memoria correspondientes a archivos de la aplicación "memorizar" de las computadoras XO.

Figura No.7

Juego de memoria de Name it

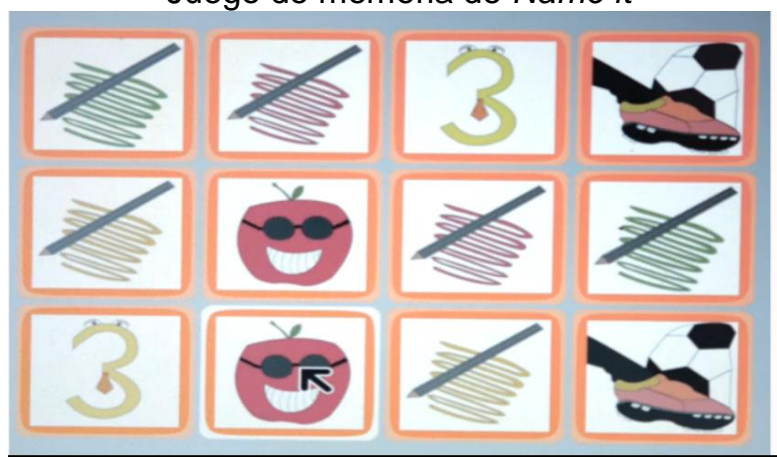

Fuente: Alfaro et al. (2015, p.17).

\subsection{Competencia ATC21S abordada}

Se espera que la utilización de los recursos de Name it faciliten a las personas docentes la realización de una actividad de aprendizaje en la que los niños y las niñas puedan tener un acercamiento transversal al desarrollo de la competencia "Comunicación", ubicada en la categoría de "Maneras de trabajar" de las compentencias ATC21S.

Name it puede contribuir particularmente a la visualización de dos indicadores. El primer indicador corresponde a la dimensión de conocimientos, y hace referencia a la adquisición de competencias en un lenguaje adicional: "Conocimiento de vocabulario básico, gramática funcional, estilo y funciones del lenguaje" (Griffin et al., 2012, p. 45). El segundo indicador corresponde a la dimensión de habilidades y hace referencia a la adquisición de competencias en lengua materna y en lenguas adicionales: "Habilidad para comunicarse, en forma oral y escrita, y comprender, o hacer que otras comprendan, varios mensajes en una variedad de situaciones y para diferentes propósitos". (Griffin et a.I., 2012, p. 45)

\section{Conclusiones}

El aprovechamiento de las computadoras XO mediante un modelo uno a uno, facilita las condiciones para la creación de ambientes de aprendizaje constructivistas, en la que los objetos de aprendizaje sustentados en tecnologías digitales pueden asumir un rol predominante en las dinámicas de clase. Según la información aportada por las personas docentes del Proyecto Conectándonos, a pesar de que los recursos audiovisuales y multimedia son utilizados con regularidad en las actividades de aprendizaje mediadas con 
las computadoras $\mathrm{XO}$, en la mayoría de éstas los recursos son usados de manera pasiva por las y los estudiantes.

Si bien este estudio no aborda directamente los factores que inciden en este aprovechamiento limitado de los objetos de aprendizaje, el autor sugiere que entre éstos se encuentra la dificultad de las personas docentes para para identificar o generar por su propia cuenta recursos audiovisuales y multimedia que puedan ser modificados, extendidos o creados por los niños y niñas, trocándose en objetos de aprendizaje. Asimismo, a pesar de que la Fundación Quirós Tanzi y el Ministerio de Educación Pública ofrecen capacitación y apoyo técnico a las personas docentes, su capacidad para diseñar y producir recursos audiovisuales y multimedia enfocadas a la mediación con computadoras XO es limitado.

En este contexto, aprovechando la información recolectada mediante las investigaciones del Centro de Investigación en Comunicación, y los recursos logísticos y humanos articulados alrededor del curso de Módulo Integrador I, se emprendió la tarea de diseñar y producir recursos audiovisuales y multimedia, perfilados como objetos de aprendizaje.

Para este fin, se construyó una metodología a través de la cual las personas productoras pudieron comprender y operacionalizar insumos teóricos relacionados con prácticas de consumo mediático y procesos de aprendizaje mediados por nuevas tecnologías en entornos de educación formal. El presente documento busca brindar pistas para que metodologías similares puedan ser replicadas en otros espacios, en donde la colaboración entre profesionales de la comunicación y profesionales de la educación pueda ser una herramienta para apoyar procesos de mediación docente.

Vale resaltar que el valor de los recursos audiovisuales y multimedia generados no se encuentra solo en la posibilidad de que sean aprovechados por la comunidad docente y estudiantil del Proyecto Conectándonos, sino también en aporte que su proceso de diseño y producción brindó a la formación profesional de las personas estudiantes participantes en el curso de Módulo Integrador. Esta contribución se vuelve particularmente importante al tomar en consideración que la Escuela de Ciencias de la Comunicación Colectivo no cuenta actualmente con cursos especializados en la construcción de recursos didácticos.

Finalmente, cabe mencionar que el Proyecto Conectándonos implementa durante el 2016 un plan piloto para distribuir y apoyar la utilización de computadoras Clamshel de Intel, con miras en un eventual relevo tecnológico de las computadoras XO como los dispositivos centrales el modelo uno a uno implementado en el Proyecto Conectándonos. De este modo, se abre una ventana para el desarrollo de investigaciones ulteriores, que elaboren sobre los 
abordajes metodológicos para contribuir a la utilización de objetos de aprendizaje en las escuelas públicas del país.

\section{Referencias}

Alfaro, Andrea, Alvarado, Melany, Barrantes, Andrey, Elizondo, Noelia, Espinoza, Tatiana, Godoy, Julián,... Soley, Virginia. (2015). Memoria de Módulo Integrador I (I-2015): Educación, Medios y Nuevas Tecnologías. [Manuscrito no publicado]. San José: Universidad de Costa Rica, Escuela de Ciencias de la Comunicación Colectiva

Badilla, Eleonora y Chacón, Alejandra. (2004). Construccionismo: Objetos para pensar, entidades públicas y micromundos. Actualidades Investigativas en Educación. 4(1), 112.

Ballestero, Jorge. (2012). Conectándonos: Un proyecto de conectividad para la educación primaria. Revista Conexiones: una experiencia más allá del aula. 4(3), 28-31.

Bloom, Benjamin. (Ed.)., Engelhart, Max, Furst, Edward y Hill, Walter, Krathwohl, David (1956). Taxonomy of Educational Objectives, Handbook I: The Cognitive Domain. New York, EEUU: David McKay Co Inc.

Colombia, Ministerio de Educación Nacional de la República. (2012). ¿Qué es un objeto de aprendizaje?. Recuperado de http://aprendeenlinea.udea.edu.co/lms/men/oac1.html

Costa Rica, Ministerio de Educación Pública y Fundación Quiroz Tanzi. (2012). Informe del proyecto Conectándonos. San José, Costa Rica: MEP.

Costa Rica, Ministerio de Educación Pública y Fundación Quirós Tanzi. (2014). Proyecto Conectándonos. Capacitación Inicial Conectándonos: una primera experiencia con ambientes de aprendizaje constructivista. San José, Costa Rica: MEP y FQT.

Costa Rica, Ministerio de Educación Pública. (2016). Matrícula inicial y final escuelas 20122016. San José, Costa Rica: MEP.

Dahl, Ole y Nygaard, Kristen. (1966). SIMULA - An algol based simulation language. Communications of the ACM. 9(9), 671-678.

Griffin, Patrick, McGraw, Barry y Care, Esther. (2012). Assessment and Teaching of $21^{\text {st }}$ Century Skills. Londres, Inglaterra: Springer.

IEEE (2002): IEEE Standard for Learning Object Metadata. Approved specifications of the IEEE. doi: 1484.12.

Instituto Nacional de Estadística y Censos. (2016). Costa Rica: Población total proyectada al 30 de junio por grupos de edades, según provincia y cantón 2016. En Estimaciones y proyecciones de población. Recuperado de http://www.inec.go.cr/poblacion/estimaciones-y-proyecciones-de-poblacion

Krathwohl, David. (2002). A revision of Bloom's Taxonomy: An Overview. Theory into Practice. 41(4), 212-218. 
Mena, Aarón. (2015). Las computadoras XO en el desarrollo de las competencias ATC21S en Costa Rica. En Vega, Patricia. III Minijornadas de investigación y acción social: Expectativas de Cambio. San José: Centro de Investigación en Comunicación, Universidad de Costa Rica

Ritzhaupt, Albert. (2010). Learning Object Systems and Strategy: A Description and Discussion. Interdisciplinary Journal of E-Learning and Learning Objects. 6, 218-238.

Uruguay, Administración Nacional de Educación Pública. (2011). Evaluación Anual en Primaria 2009-2011. Montevideo, Uruguay: ANEP.

Wiley, David. (2000). Connecting learning objects to instructional design theory: A definition, a metaphor, and a taxonomy. Recuperado de http://reusability.org/read/chapters/wiley.doc 